\title{
Smart Gate Pass Security Management System Using Random Key Generation
}

\author{
Richita Rodrigues ${ }^{1}$, Aruna Pavate ${ }^{2}$, Rujuta Sawant ${ }^{3}$ and Nehal Lopes ${ }^{4}$ \\ ${ }^{1,3,4}$ Student (Under Graduate), Department of Computer Science \& Engineering, St. Francis Institute of Technology College \\ of Engineering, Mumbai , Maharashtra, India \\ ${ }^{2}$ Assistant Professor, Department of Computer Science \& Engineering, St. Francis Institute of Technology College of \\ Engineering, Mumbai , Maharashtra, India
}

Correspondence should be addressed to Richita Rodrigues; rodriguesrichita@ gmail.com

Copyright () 2021 Made Richita Rodrigues et al. This is an open access article distributed under the Creative Commons Attribution License, which permits unrestricted use, distribution, and reproduction in any medium, provided the original work is properly cited.

\begin{abstract}
Gate pass management system relates to the act of security. This system can be used to speed up the visitor registration which will notify the presence of visitors in the organisation. This can be used to replace the traditional visitor registration process. This reduces the problem of appointments and gathering information about the visitor via web and android application and this information is recorded in the database that manages the data. The Gate pass Management System (GMS) organises the visitor record and reduces the time of management and enhances the level of security.
\end{abstract}

KEYWORDS- OTP, Android Application, Web Application, Gate, Admin and Visitor.

\section{INTRODUCTION}

Nowadays, most of the organisations are using the register method to keep track of incoming and outgoing visitors record of each department. This creates a problem when the number of visitors visiting the department increases and are unable to manage the records. One of the main issues in generating the visitor pass of the organisation is security. Gate Pass Management System (GPMS) also helps to access the user information faster [1]. In the manual system, the user needs to search the information page by page. However, with the new system, users only need to enter details in an android application which provides access to the gate only after authentication and this system also displays the details that have been entered by the user. The user can book appointments prior to the visit through the website. The problem of waiting in a long queue is reduced. GPMS help to search the visitor details easily. A notification is sent to the system about the visitor entering the department. Searching is done at a faster rate and the result is generated. The Gate Pass Management System (GPMS) provides an easy approach to keep track of the entry and exit of a visitor by taking inputs like phone number, email and personal details. The system aims to secure the company from outside visitors by first taking prior details from the visitor and booking an appointment through a web application. The admin grants or rejects the appointment requested by the visitor. After granting the appointment the details which are entered are stored in the database by the admin. When the visitor visits the company he has to enter email id, phone number and other details in an android application. The email id is cross checked with the stored email id in the database. If the email id is verified then the visitor receives random generated code on his email which has to be entered in the android application. It acts as an access to the gateway. As soon as a visitor enters the entry time is saved in the database and the door gets automatically locked thus preventing multiple entries. The system is again set to accept a new visitor.

At the exit point the email is taken as input and again a new random key is assigned to the user. As soon as a visitor exits the exit time is saved in the database and the door gets automatically locked thus preventing multiple exits. The admin can access the details of the visitor from the database. The details of visitors would be saved into a database and the admin grants the permission. The visitor gets a confirmation that he is allowed to enter the organisation. This system proposes the "Paper Saving Idea". The detailed working of the work is organized as section 2 gives related work with the current work, section 3 concentrates on the Methodology applied, section 4 discusses results and analysis, section 5 concludes the work.

\section{RELATED WORK}

Several papers have been referenced related to this topic which is written in [1] The authors H. Gunawan and E. A. Kadir has implemented the student system which has information and student identity. Student information system uses computer networking to update every transaction and then send the data to the system. Every record and transaction of a student passing the gate will be monitored by the automatic gate system. Another research was to reduce the use of paper work. The authors in the paper aim to develop a system using Raspberry Pi for the gate pass system. The main aim was to save paper by sending SMS and Email for verification of users with the help of Internet Connectivity. The Visitor Information Management System enables capturing new visiting records [2]. The information of the visitor is recorded in a centralized database server. This provides data management and manipulation of the records through searching the 
report. Now-a-days everything is based on touch screens like our smartphones, laptops, smart TV's, etc. It becomes easy to give input using touch and also the output is received on it. Touchscreen operation is very easy and can be done by people of any age. Touch screens here have a very important role in getting data from students to generate his/her gate pass [3]. To save paper authors have generated this automated system for a gate pass where fast and accurate work can be done and the usage of paper comes to null. In this paper they are trying to design a runtime monitoring system. For this a good internal feedback system and communication is required between devices [4]. In this paper the authors R. Halemani and A. Rajagopal has proposed a low cost interface to control electrical loads that also provides security of building, residential or commercial, for the user in terms of development, operation and easy to use interface is presented. The various devices can communicate over CAN bus and the nodes can be controlled remotely via a gateway, which is connected to the internet. The significance of this topic is related to society and the specific topic discussed is Building Automation and Security Using CAN And IOT. The things referred to can change from a variety of devices like chip transducers in animals, built-in sensors in automobiles, fire assistant devices for fire fighters, washing machines, thermostats, etc[5].Human Locomotion by using feet tracking allows a person to track objects like limbs and head. This is done by temporarily locking the position alternately to the user's feet The main aim of this approach is to reduce costs for tracking human locomotion [8]. The next is the poster abstract which is to track people's movements using low cost and non-sensor equipped BLE Beacons. This system tracks information of people in buildings, organisations and hospitals. From this we will come to know in which area people are present and the system is low cost and easy to install and maintain [9]. Intruder tracking can be done by using wireless sensor networks which develop an application that identifies moving objects using various sensors. This system is used to detect the motion of unauthorised people. Target tracking, data processing and analysis is a major component of this type of application. The advantage of this system is that it does not require any existing infrastructure [10].

\section{METHODOLOGY}

The proposed system utilizes the network concepts to deal with the out-pass process in the office. The system simplifies the process by transferring the gate pass through by first taking details like email id and phone number from the visitor via an android application which generates a random key which can be used only once. The visitor will receive random generated code on his mobile via E-mail which has to be entered in the android application and it will act as an access to the gateway. So the person can't edit the data in the gate pass. The system also helps to store and track the time log easily. Generating a random key is an emerging field of research that has many challenges such as large sets of numbers, noisy data, improper illuminating conditions etc., Eigen random key approach helps to overcome these obstacles in developing a system for generating a random key, in the gate pass generation in offices for visitors. In this proposed system, we propose a new software tool for random key generation and pass generation for office campus. This application is fully applied with the visitor's pass. Once the person wants the out pass then at the exit point the email id is be taken as input and again a new random key will be assigned to the user. As soon as a visitor exits the exit time is saved in the database and the door will get automatically locked thus preventing multiple exits. The main aim of this system is to provide gate pass security to the office campus.

The authorized person will be allowed by the system to leave from the campus and if the person is found to be authorized then it proceeds for the generation of gate pass with effective rule matching. This system is divided in two parts, the random key generation system and the generation of the gate pass for the authorized person.
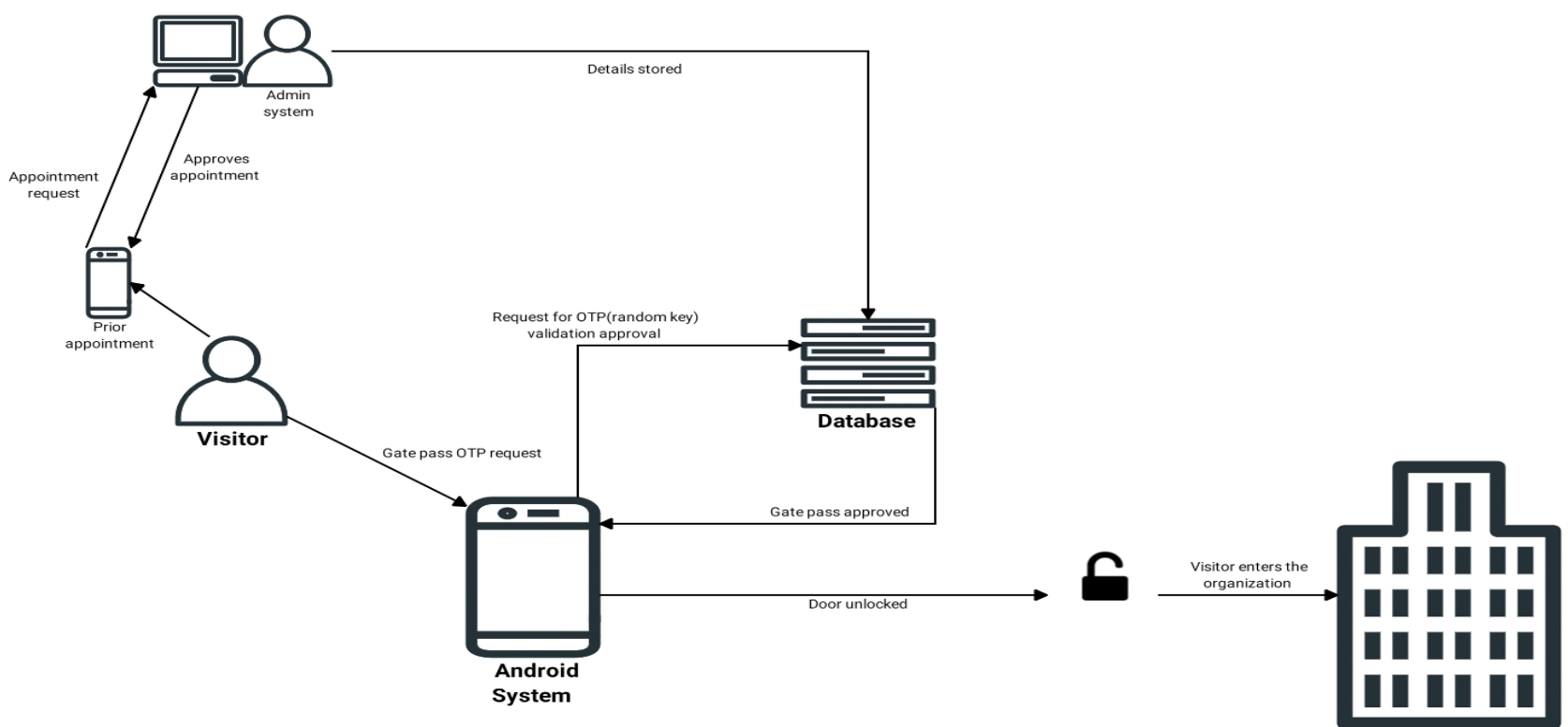

Fig. 1: Proposed block diagram for Gate Pass Security Management 
Figure 1 represents a block diagram of the Gate Pass Security System in which the visitor enters his details into the website. A request is sent to the admin and after the request is granted, the visitor enters his details in the android system. An OTP is received on his email address which has to be entered in the android application and the gate opens if the otp is correct.

\section{RESULT AND ANALYSIS}

The goal of our project is to build a security gate pass for visitors so that they can enter the company's premises with prior appointment. This includes user-friendly web applications, an android application and system hardware. Thus a system named 'Gate Pass Management System Using Random Key Generation’ is developed.

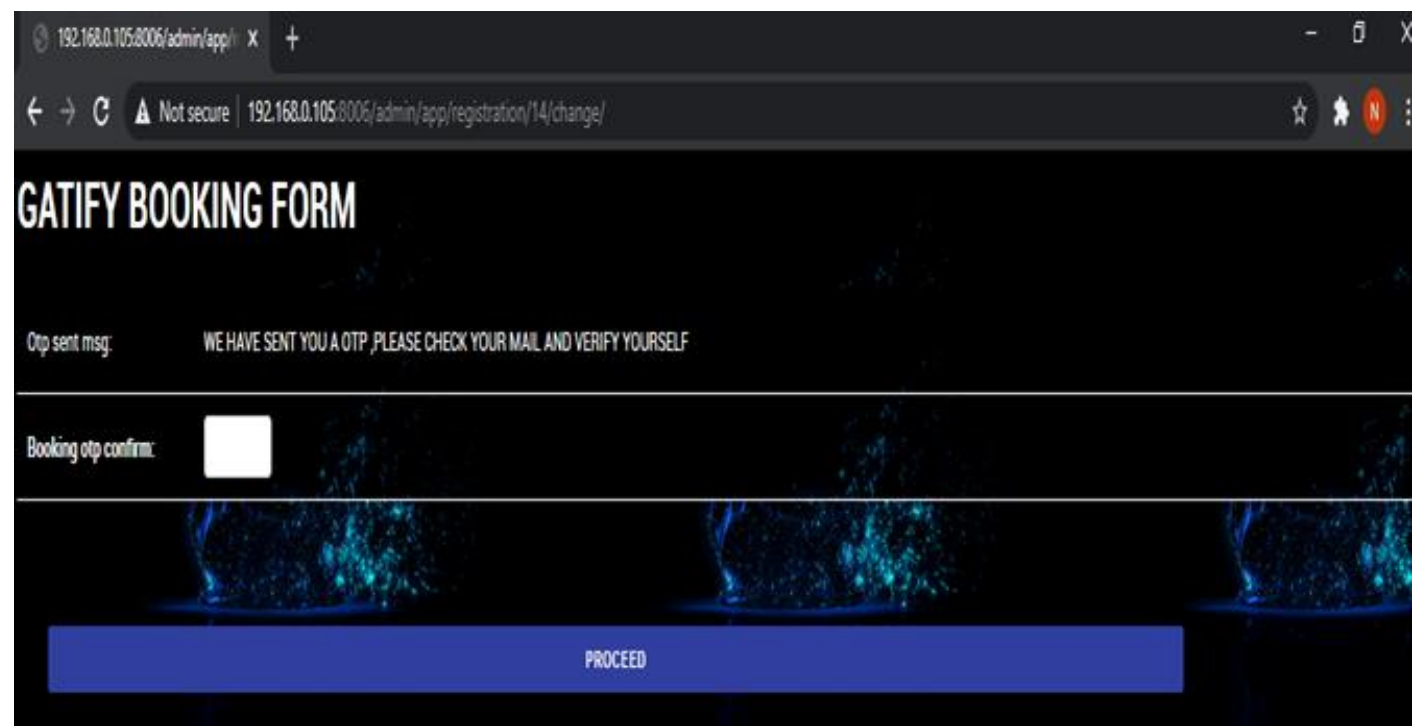

Fig. 2: Booking Confirmation Page

Figure 2 represents a booking confirmation page where an OTP sent message is displayed.

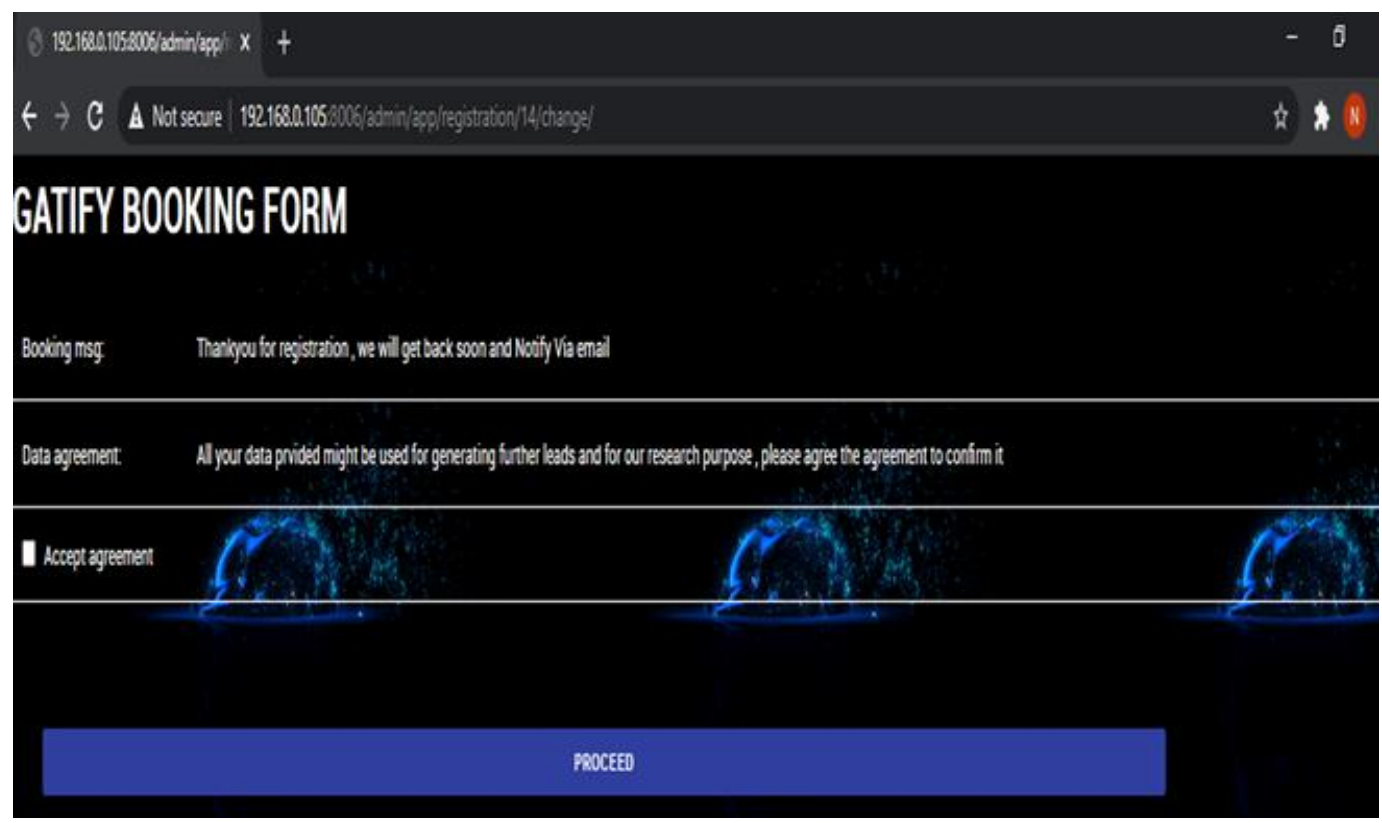

Fig. 3: Policy / Agreement Page

Figure 3 displays a booking confirmation message along with an acceptance of data agreement which the organisation uses for its future process. 


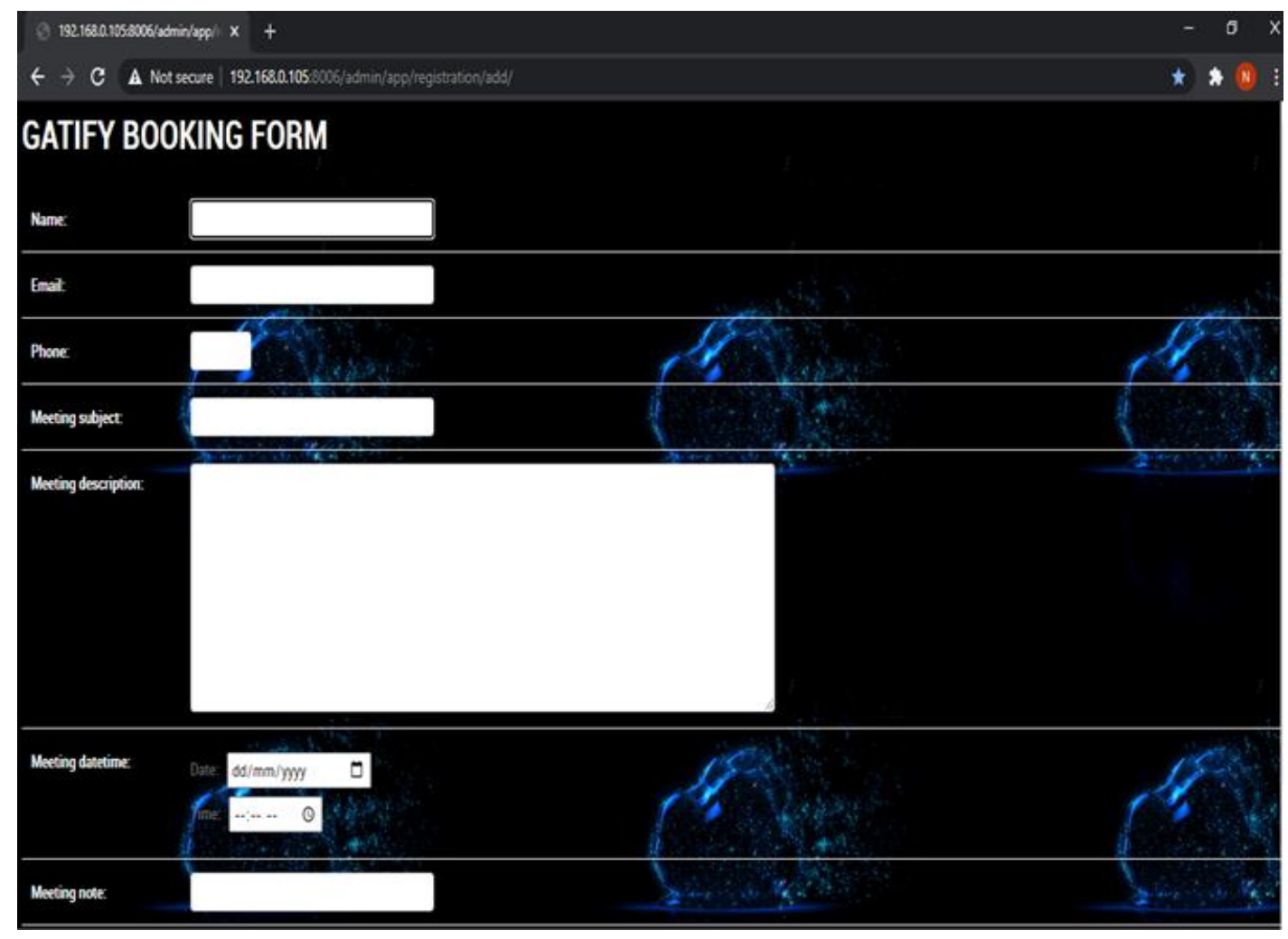

Fig. 4: Visitor Registration Page

Figure 4 represents that the visitor has to prior book his/her appointment by visiting the organisation's registration page.

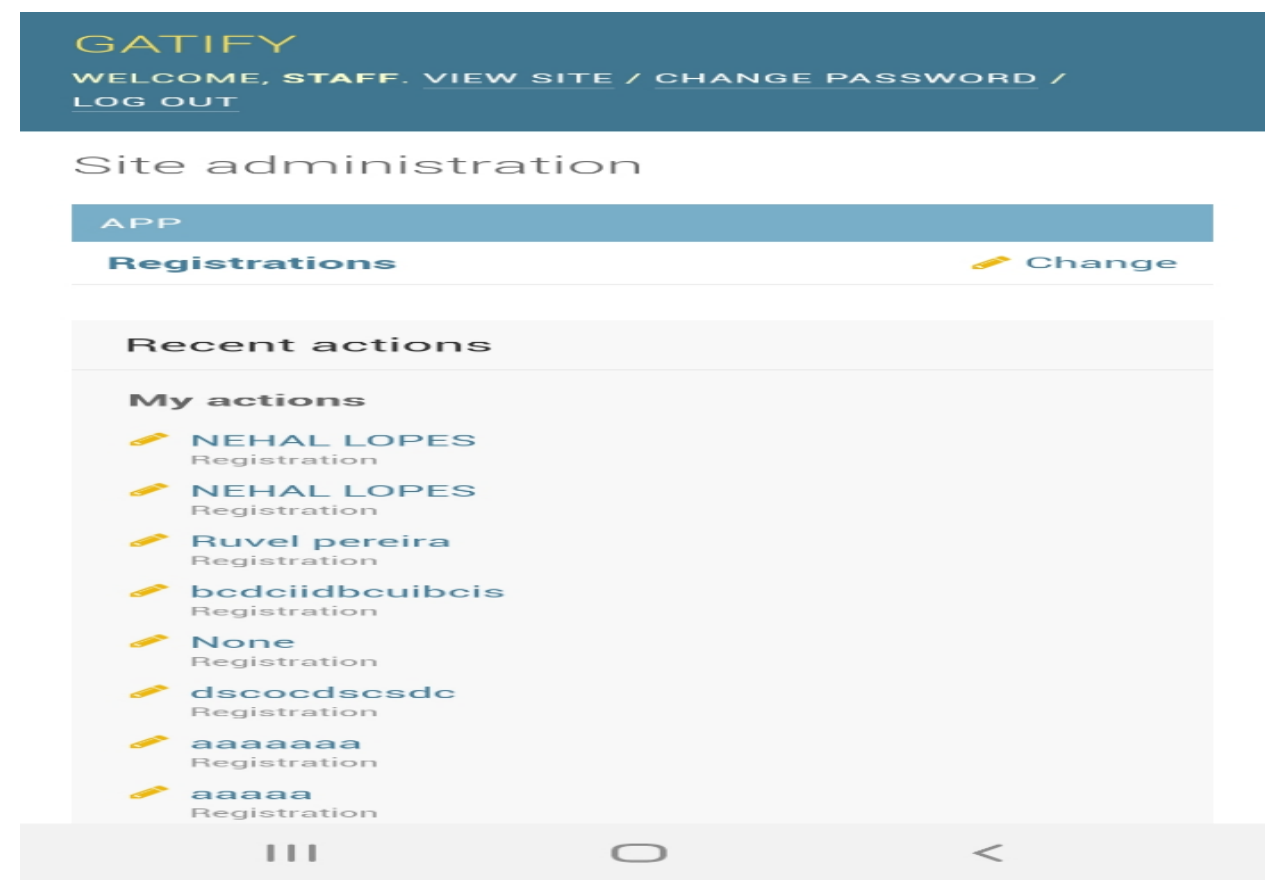

Fig. 5: Home Page

Figure 5 represents the Home Page of the application where we can see recent actions, also find out registration done by visitor using booking id.

The system includes a web application where the visitors can do registration before visiting the company. The registration names are shown in the dashboard of the website. A booking id is created for each registration and the list is displayed on the web page. We can search a particular registration by entering a particular booking id. 

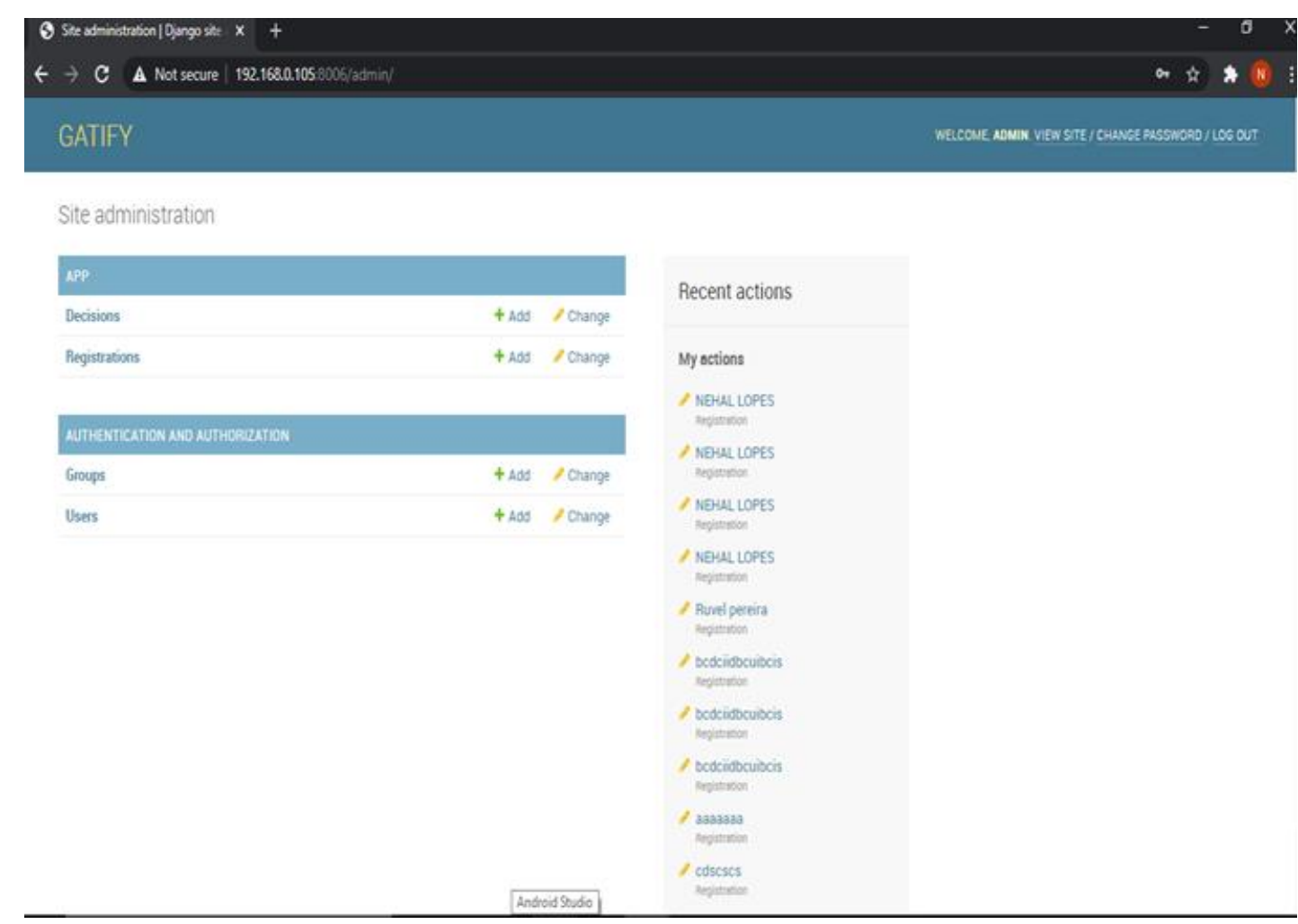

Fig. 6: Admin Dashboard Page

As shown in the figure 6 , we can add new users, registrations, decisions and groups. The admin can edit, delete or change the details of the visitors.

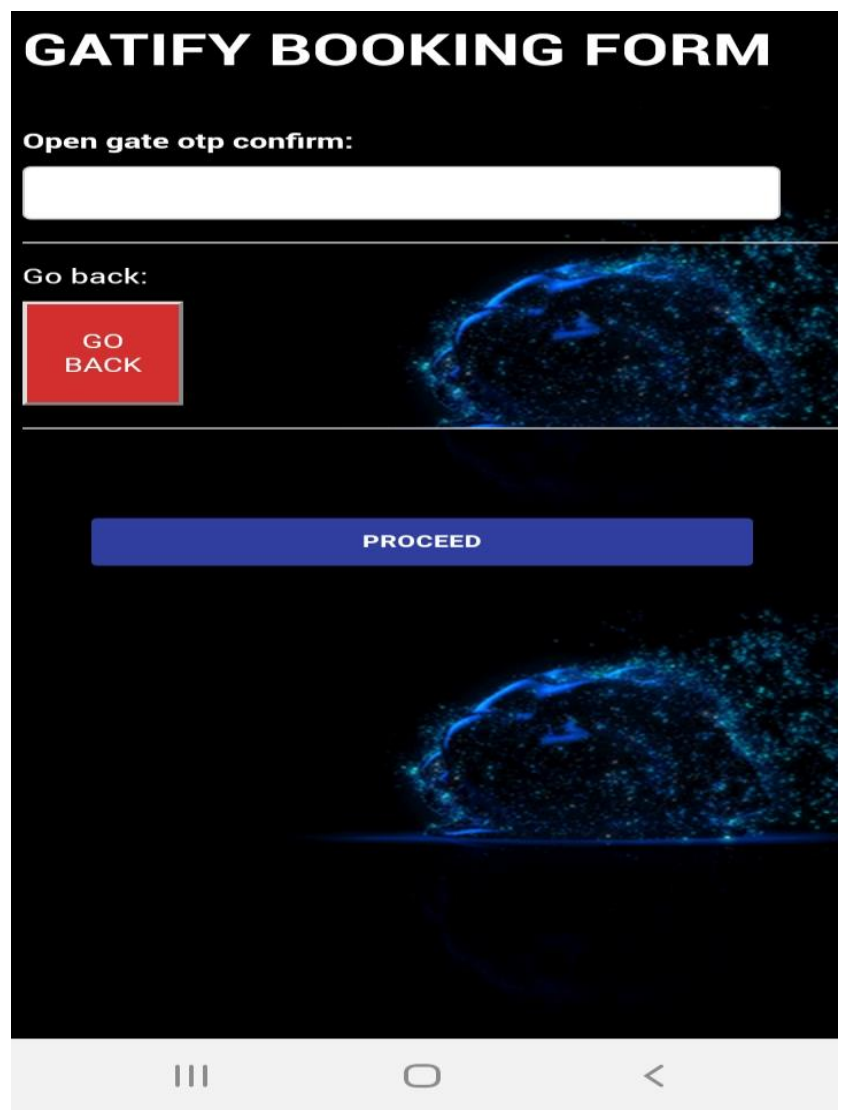

Fig. 7: Gate OTP Verification
As shown in the figure 7 , a visitor has to enter the received OTP and click on the proceed button. The visitor can also press the go back button to go to the previous page of the application.

\section{CONCLUSION}

The GPMS increases the level of security of an organisation. GPMS provides a way of securing visitor registration. The records in the computer provide better data manipulation and management. Its installation is easy and does not require any expert. The GPMS system is easy to use and maintain. The system is reliable and provides security. This restricts the users who don't have authority from entering the organisation. Biometric can also be used along with the system to increase the level of security. Hence this system provides an efficient system to obtain a gate pass for a particular organization. This decreases the paper consumption and it proves to be a more secure and easy process that helps to save time. Visitor gate pass management system provides a way to control and track your organization's visitor traffic. It prevents the entry of users who do not have authority to enter the organisation. The architecture of the visitor gate pass management System allows various people which are authorized in the organization to directly access the data. This system security of the premises of an organisation is enhanced. The data of an organisation for any purpose is stored and processed by the computer. The data can be accessed and processed at a faster pace. Therefore, the GPMS system will be able to solve various problems. The level of security in the premises of the organizations is increased to a greater level. GPMS provides a secure way of visitor registration. The records in the computer provide better data manipulation and management. Its installation is easy and 


\section{Smart Gate Pass Security Management System Using Random Key Generation}

does not require any expert. This can save time in obtaining gate pass and make procedures easier and faster. The admin generates the report directly from the database rather than maintaining the record of gate pass taken by each visitor "n" number of times.

\section{REFERENCES}

[1] H. Gunawan and E. A. Kadir, "Integration protocol student academic information to campus RFID gate pass system," 2017 4th International Conference on Electrical Engineering, Computer Science and Informatics (EECSI, Yogyakarta, 2017, pp. 1-6.

[2] Harish Rapartiwar, Pushpanjali Shivratri, Omkar Sonakul, Prof. Ashwini Bhugul Computer Engineering, Marathwada Mitra Mandal's College of Engineering, Pune, India, Visitor Gate Pass Management System : IJCSMC, Vol. 6, Issue. 2, February 2017.

[3] Prof. Abhay Gaidhani1, Suraj Sahijwani2, Parag Jain3, Shantanu Jadhav4, Ankush Jain5 1,2,3,4,5 Department of Computer Engineering, Sandip Institute of Engineering Management, Nashik, Volume 02, Issue 10, [October 2015].

[4] Mallesham Yerragolla, Kamalakar Pallela, Indira Priyadarshini Gera, " 2016 IEEE Uttar Pradesh Section International Conference on Electrical, Computer and Electronics Engineering (UPCON) Indian Institute of Technology (Banaras Hindu University) Varanasi, India, Dec 9-11, 2016.

[5] R. Halemani and A. Rajagopal, "Building automationsecurity using can and IoT," 2015 International Conference on Applied and Theoretical Computing and Communication Technology (iCATccT), Davangere, 2015, pp. 471-476.

[6] S. Sivathasan, "RF/FSO and LEACH wireless sensor networks: A case study comparing network performance," 2009 IFIP International Conference on Wireless and Optical Communications Networks,Cairo, 2009.

[7] IEEE Health informatics-PoC medical device communication Part 00101: Guide- Guidelines for the use of RF wireless technology," in IEEE Std 11073-00101-2008 ,vol., no., pp.1-125, 26 Dec. 2008.

[8] H. Tsai, G. Chen and H. Lee, "Poster Abstract: Using LowCost, Non-Sensor-Equipped BLE Beacons to Track People's Movements," 2017 16th ACM/IEEE International Conference on Information Processing in Sensor Networks (IPSN), Pittsburgh, PA, 2017, pp. 291-292.

[9] R. C. Jisha, M. V. Ramesh and G. S. Lekshmi, "Intruder tracking using wireless sensor network," 2010 IEEE International Conference on Computational Intelligence and Computing Research, Coimbatore, 2010, pp. 1-5.

[10] M. Zank, T. Nescher and A. Kunz, "Tracking human locomotion by relative positional feet tracking," 2015 IEEE Virtual Reality (VR), Arles, 2015, pp. 317-318.

\section{ABOUT THE AUTHORS}

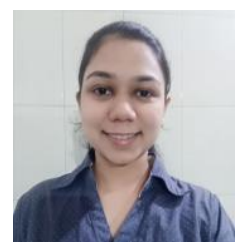

Richita Rodrigues is currently a final year student who is pursuing a Bachelor of Engineering degree in Information Technology from Mumbai University. Her research interests include web development and IOT.

Rujuta Sawant is currently a final year student who is pursuing a Bachelor of Engineering degree in Information Technology from Mumbai University. Her research interests include Android Development, Web development and IOT.

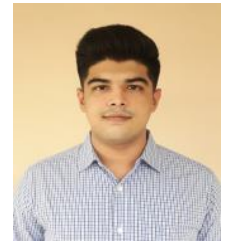

Nehal Lopes is currently a final year student who is pursuing Bachelor of Engineering degree in Information Technology from Mumbai University. His research interests include web development, android development and IOT .

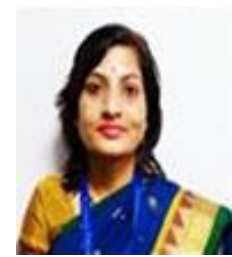

Aruna Pavate pursuing her Research in Information Technology from Mumbai University and working as an Assistant Professor at SFIT college of Engineering, Mumbai, India. Currently working on new advances in the field of medical and engineering to improve in the healthcare domain. Her research interests include Machine learning and security, data mining, Data Science. She also spent a few years working in the industry. She has over 40 peer reviewed articles. She is an editorial board member, reviewer for Science publishing group 2020, and Guest editor of special Issue on Advanced deep learning methods for Audio and Speech Processing. She had worked as reviewer for Journal American Journal Experts (AJE) as well as worked as reviewer for many conferences. She had been Program Chair for IFERP Unit of VVERT Conference 2015 \& 2016, Mumbai India. She is having member of professional bodies ISTE, IAENG, AICTSD,Insc 